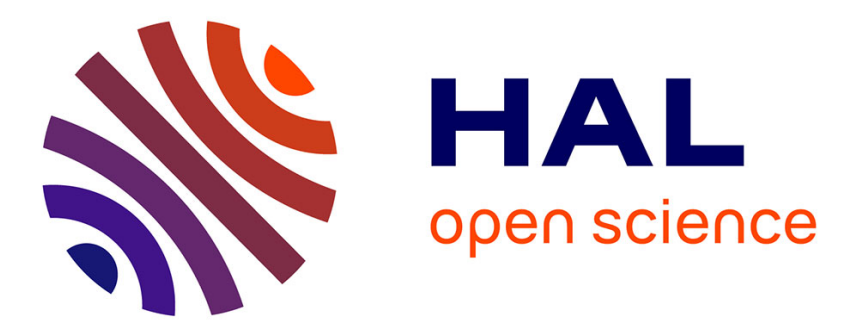

\title{
High resolution photoemission spectroscopy of flat and stepped non reconstructed $\mathrm{H} / \mathrm{Si}(111)$ surfaces
}

A. Taleb-Ibrahimi, R. Günther, P. Dumas, G. Indlekofer, Y. Chabal, Y. Petroff

\section{To cite this version:}

A. Taleb-Ibrahimi, R. Günther, P. Dumas, G. Indlekofer, Y. Chabal, et al.. High resolution photoemission spectroscopy of flat and stepped non reconstructed $\mathrm{H} / \mathrm{Si}(111)$ surfaces. Journal de Physique IV Proceedings, 1994, 04 (C9), pp.C9-89-C9-95. 10.1051/jp4:1994911 . jpa-00253471

\section{HAL Id: jpa-00253471 https://hal.science/jpa-00253471}

Submitted on 1 Jan 1994

HAL is a multi-disciplinary open access archive for the deposit and dissemination of scientific research documents, whether they are published or not. The documents may come from teaching and research institutions in France or abroad, or from public or private research centers.
L'archive ouverte pluridisciplinaire HAL, est destinée au dépôt et à la diffusion de documents scientifiques de niveau recherche, publiés ou non, émanant des établissements d'enseignement et de recherche français ou étrangers, des laboratoires publics ou privés. 


\title{
High resolution photoemission spectroscopy of flat and stepped non reconstructed $\mathrm{H} / \mathrm{Si}(111)$ surfaces
}

\author{
A. Taleb-Ibrahimi, R. Günther, P. Dumas (1), G. Indlekofer, Y.J. Chabal* and Y. Petroff** \\ LURE, Bat 209D, Centre Universitaire Paris-Sud, 91405 Orsay cedex, France \\ * ATT Bell Laboratories, 600 Mountain Avenue, NJ 07974 Murray-Hill, U.S.A. \\ ** European Radiation Synchrotron Facility, 38000 Grenoble, France
}

\begin{abstract}
Chemically prepared, long range ordered, ideally hydrogen terminated $\mathrm{Si}(111)$ surfaces are model systems for the study of the electronic properties of silicon surfaces. The perfection of these surfaces leads to very sharp features in the high resolution photoemission spectra. New theoretical approaches based on first principle calculations agree with our experimental results, which allows a better understanding of the origin of the surfaces states at the $\mathrm{H} / \mathrm{Si}(111)$ surfaces. The chemical treatment of vicinal Si(111) surfaces leads to dihydride or monohydride stepped surfaces. The comparison between flat and stepped surfaces show that long range order defects do not induce any important broadening of the spectral features. However additional surface states in the valence band spectra of the dihydride terminated surfaces are observed which are probably due to a local deformation at the steps.
\end{abstract}

\section{Introduction}

The electronic properties of clean silicon surfaces have been extensively studied in the past. Due to the variety of reconstructions, characteristic of clean semiconductor surfaces, the near-surface strains mainly contribute to the spread of experimental values obtained in photoemission, in both the valence band and core level regions. The importance of local ordering in the number, position and lineshape of the surface states, in both energy ranges, has been recently adressed by comparison of the photoemission data obtained on ideally hydrogen terminated, non reconstructed $\mathrm{Si}(111)$ surfaces, with those obtained on clean and reconstructed $\mathrm{Si}$ surfaces [1].

The non reconstructed, ideally hydrogen terminated $\mathrm{Si}(111)$ surfaces are obtained by a wet chemical treatment in buffered HF solutions[2], and by their carefull introduction into an ultra high vacuum environment (UHV) precluding hydrogen loss and contamination[3]. The simplicity and high degree of order of these surfaces have motivated new theoretical studies, for the valence band[1] and the core level states[4], based on first principles calculations using a quasiparticle self energy approach. These studies give unravelled agreement with the experimental data.

Moreover, the aqueous fluorine etching of vicinal $\mathrm{Si}(111)$ surfaces leads to the formation of extremely straight steps distributed in a regular array with edges terminated by monohydrides or dihydrides, depending on the orientation of the cut out of the (111) plane[5]. The type, concentration and geometry of these defects being very well controlled, stepped surfaces produced by this chemical treatment can be regarded as model systems for the study of the influence of known defects on the electronic properties of silicon surfaces.

In this paper we first report on valence band and core level ARPES spectra of ideally dihydride terminated stepped $\mathrm{Si}(111)$ surfaces and compare them to the results obtained on flat surfaces for equivalent photoelectron energies and emission angles. 
In the second part of the paper we discuss the core level shifts observed for a flat hydrogen terminated $\mathrm{H} / \mathrm{Si}(111)$ surface in the light of a new theoretical approach[4]. We show that there is a very satisfactory agreement between our experimental data and the calculated ones of Blase et al.

\section{Experimental details}

\section{Instrumentation}

The spectra are measured using the high resolution photoemission French-Swiss beamline (SU3) at SuperAco, in LURE. This undulator beam line is equipped with a $9 \mathrm{~m}$ plane grating monochromator (PGM). A high resolution HA150 VSW hemispherical analyser is used for the detection of the photoemitted electrons. A total resolution of $25 \mathrm{meV}$ is achieved for $17 \mathrm{eV}$ photon energy and $45 \mathrm{meV}$ for $110 \mathrm{eV}$.

\section{Sample preparation}

The silicon samples ( As-doped, $\rho=100 \Omega \mathrm{cm}$ ) are first thermally oxidized with a 15 min post anneal at $1050^{\circ} \mathrm{C}$. Vicinal surfaces are cut $9^{\circ}$ off the (111) plane, in the $\langle\overline{1} 12\rangle$ direction for the dihydride terminated steps. For a perfect array of equal length terraces, this arrangement would involve 6 terrace atoms, with an average terrace length of $19.8 \AA$ along the $\langle\overline{1} \overline{1} 2\rangle$ direction.

Flat and stepped surfaces are degreased in hot trichloroethylene, then in hot acetone and methanol. They are subsequently cleaned in a solution of $\mathrm{NH} 4 \mathrm{OH}: \mathrm{H} 2 \mathrm{O} 2: \mathrm{H} 2 \mathrm{O}$ at $80^{\circ} \mathrm{C}$. The final etching step consists of dipping the samples in an ammonium fluoride solution followed by thorough rinsing in deionised water $(18.2 \Omega \mathrm{cm})$. The introduction procedure of the samples in the UHV chamber was previously described[3].

\section{Results and discussion}

In the following, we present spectra obtained for flat and dihydride terminated stepped $\mathrm{H} / \mathrm{Si}(111)$ samples in the valence band and in the core level regions, respectively.

\section{Valence band region:}

A series of angle-resolved photoemission spectra, taken at $17.5 \mathrm{eV}$ incident photon energy on flat $\mathrm{H} / \mathrm{Si}(111)-(1 \times 1)$ surface, in the vicinity of the $\overrightarrow{\mathrm{K}}$ point of the Surface Brillouin Zone (SBZ), is displayed on Fig.1. The high degree of homogeneity of the surface is inferred by the remarkably narrow width of the surface states observed ( less than $330 \mathrm{meV}$ ) compared to those obtained on atomic $\mathrm{H}$ exposed $\mathrm{Si}(111)$ [6], or on ideally $\mathrm{H}$ terminated $\mathrm{Si}(111)-(1 \times 1)$ obtained by removal of an indium layer by atomic hydrogen[7]. The position, dispersion and pocket size of the valence surface states are in a very satisfactory agreement with a theory based on first-principle calculations using a quasiparticle self-energy approach within the GW approximation [1]. The peaks, labelled (a) and ( $\left.\mathrm{a}^{\prime}\right)$ are assigned to the Si-H bonds and the backbonds respectively.

Valence band spectra were also recorded on stepped $\mathrm{H} / \mathrm{Si}(111)$ samples. The photoelectrons were again collected for different emission angles, but also for different orientations of the dihydride steps with respect to the analyser. The collected photoelectrons originate from different surface atoms (terrace atoms, step edge atoms or foot atoms ) depending on whether the emission plane is parallel or perpendicular to the step edge [110] direction. Fig. 2 displays electron distribution curves for a dihydride terminated surface taken at $17.5 \mathrm{eV}$ photon energy, with an emission angle of $55^{\circ}$ for three orientations of the steps with respect to the detector, one orientation corresponds to the emission plane parallel to the steps, and the two others to an emission plane perpendicular to the steps: upward (steps going up) and downward (steps 
going down). The spectra presented here correspond to a fixed value of the parallel momentum. The band dispersions, and a more complete analysis of the valence band spectra of stepped $\mathrm{H} / \mathrm{Si}(111)$ surface will be presented elsewhere[8].

The most interesting observation is the presence of a surface state, indicated by an arrow, located at $0.8+1-$ $0.1 \mathrm{eV}$ below the Fermi level, when the photoelectrons of the lower end of the dihydride steps (downward orientation) also contribute to the detected signal. This surface state does not exhibit any detectable dispersion[8]. In the opposite direction (upward steps) and in the case where the emission plane is parallel to the steps, such a state is not observed. Therefore, this new feature, compared to the valence band structure of the flat surface, should originate from a particular defect, or strain, located at the lower end of the dihydride steps. It has been shown by Raman spectroscopy[5], that strong steric interaction exists between the lower $\mathrm{H}$ atom of the vertical dihydride, and the closest $\mathrm{H}$ atom of the lower terrace (see insert Fig2). Consequently, the dihydride unit was found to rotate backward from the terrace by $22^{\circ}$, both theoretically [9] and experimentally[10], in order to relieve some of the steric hindrance. The surface state at $-0.8 \mathrm{eV}$, below the Fermi level, is observed only when photoelectrons originating from the lower end of the steps are not shadowed by the step itself. We thus suggest that some local deformation at the steps, is responsible for this surface state.

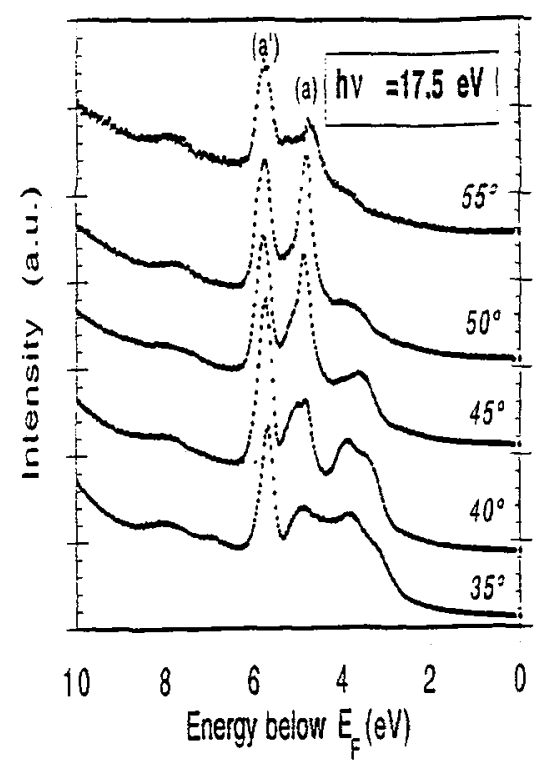

Fig.1: Angle resolved photoemission spectra, recorded on flat $\mathrm{H} / \mathrm{Si}(111)-(1 \times 1)$ surfaces with a $17.5 \mathrm{eV}$ incident photon energy. Energy and angle resolution are 25 $\mathrm{meV}$ and $+i-1^{\circ}$ respectively. The photons are incident at grazing incidence, with a projection along the $<112>$ direction, and the photoelectrons are collected in a plane perpendicular to the incident plane probing along the $\bar{\Gamma} \overline{\mathrm{K}}$ direction.

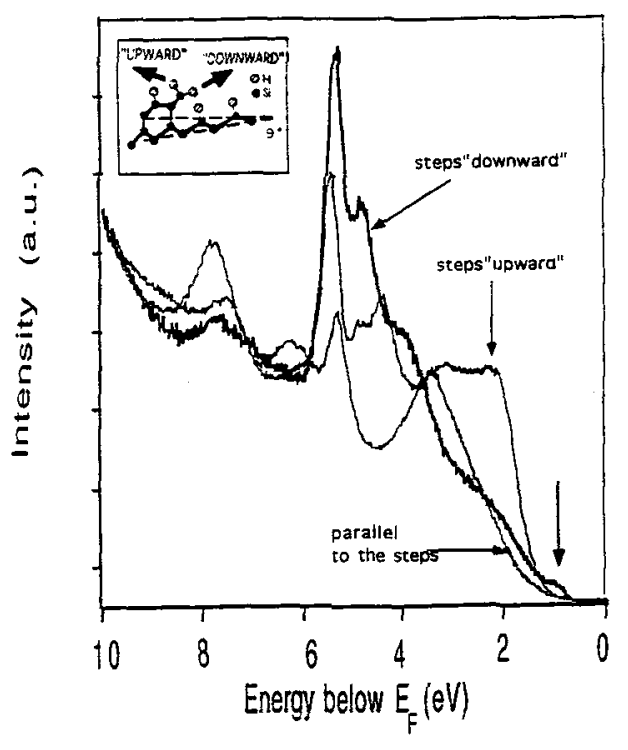

Fig. 2: Valence band spectra obtained on a dihy dride terminated stepped $\mathrm{H} / \mathrm{Si}(111)$ surface. While the emission angle is fixed to $55^{\circ}$. the spectra correspond to three orientations of the steps with respect to the analyser : the emission plane parallel to the steps, the emission plane perpendicular to the steps with the steps going up toward the analyser (upward direction as sketched in the insert) or going down (downward direction). The incident photon energy is $17.5 \mathrm{eV}$. 


\section{Core level region:}

Long range ordered $\mathrm{H} / \mathrm{Si}(111)-(1 \times 1)$ surfaces exhibit exceptionally narrow features in the core level region[1]. However, slight changes of the width of the different components are observed, depending on the quality of the chemically prepared sample, i.e. of the extent of its long range ordering thus emphasizing the role of the sample preparation. .

Fig 3 displays the core level spectra of a flat $\mathrm{H} / \mathrm{Si}(111)$ surface for $130 \mathrm{eV}$ (surface sensitive) photon energy, at $300 \mathrm{~K}$ and $100 \mathrm{~K}$ ( the lower temperature should be considered with a large uncertainty, as the thermocouple was not directly attached to the movable sample holder). A net change of the intensity ratio between the bulk component and the surface component is noticeable as a function of temperature showing the temperature dependence of the surface and bulk phonon broadening ( see Table 1 for phonon broadening values). This is also clearly seen on bulk sensitive spectra, taken at $108 \mathrm{eV}$ incident photon energy (Fig4).

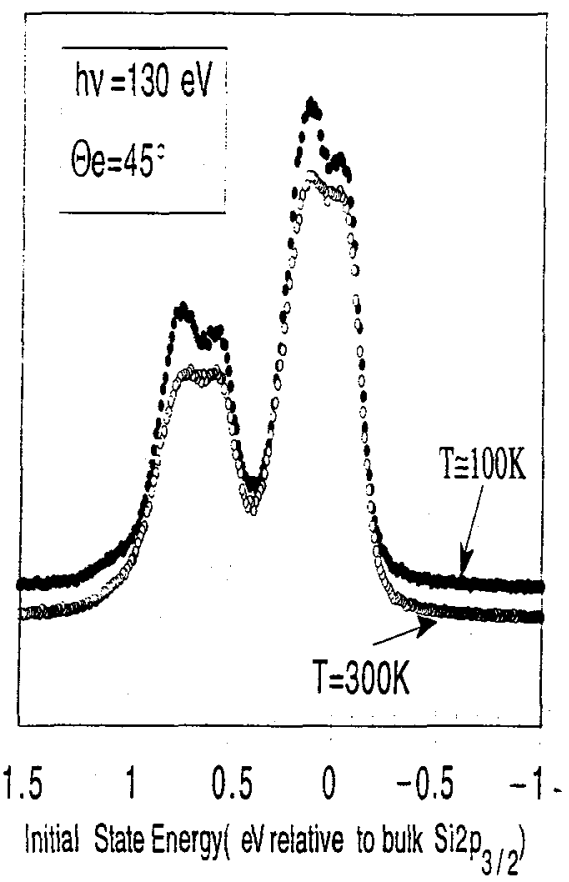

Fig.3: Comparison of surface sensitive Si2p core level spectra taken on a flat $\mathrm{H} / \mathrm{Si}(111)-$ (1x1) surface, recorded at two different temperatures. The photon energy is $130 \mathrm{eV}$, and the emission angle is $45^{\circ}$. The two spectra are offset for clarity. Note the change in the relative intensity between the bulk feature and the surface feature located at $-140 \mathrm{meV}$.

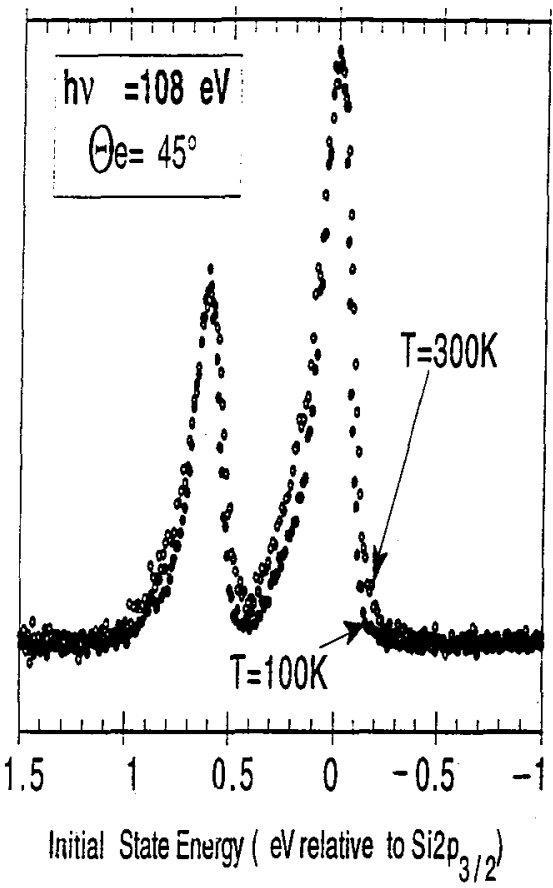

Fig.4: Comparison of bulk sensitive Si 2pcore level spectra taken on a flat $\mathrm{H} / \mathrm{Si}(111)-(1 \times 1)$ surface, recorded at two different temperatures. The photon energy is $108 \mathrm{eV}$, and the angle of emission is $45^{\circ}$.A net narrowing of the bulk components is observed.

In order to obtain quantitative values for the energy position, number and width of the surface states, curve fittings are performed, using Voigt functions. The procedure used is the same as the originally published one[1], but the fitting parameters are adjusted in the light of recent calculations of the Si $2 \mathrm{p}$ core level shifts for atoms near the H/Si(111) surface by Blase et al. [4]. The authors show that the use of first order perturbation theory in order to describe core-valence interactions in the frame of an ab initio pseudopotential approach gives very good results for the Si2p core level shifts on the H/Si(111) surface. 
They find, that the $2 \mathrm{p} 3 / 2$ core level of a silicon atom at the surface is split into two peaks, $S_{1=2 \mathrm{p} 3 / 2^{(-)}}$ and $S_{2}=2 \mathrm{p}_{3 / 2}{ }^{(+)}$on the higher binding energy side of the bulk core level, due to crystal field effects. They calculated this splitting to be $70 \mathrm{meV}$, and their relative intensities which depend strongly on the polarisation of the incident photons. On the other hand no splitting is expected for the Si $2 \mathrm{p} 1 / 2$ line on the ground of group theory analysis. In addition, the subsurface silicon atom component (backbonds) is calculated to be at lower binding energy which agrees with the previous experimental findings[1]. Our curve fitting procedure takes into account the theoretically predicted parameters and the crystal field effect. Fig. 5 displays a Si2p core level spectrum, recorded in a surface sensitive mode (hv=130 eV) with the incident electric field parallel to the surface (s polarisation), together with the resulting deconvolution. In the fitting procedure we imposed the intensity ratio between the two surface $\mathrm{Si} 2 \mathrm{p} 3 / 2$ components to be equal to the theoretically predicted one $(S 1 / S 2=0.4$ for a s-polarised photon beam ).

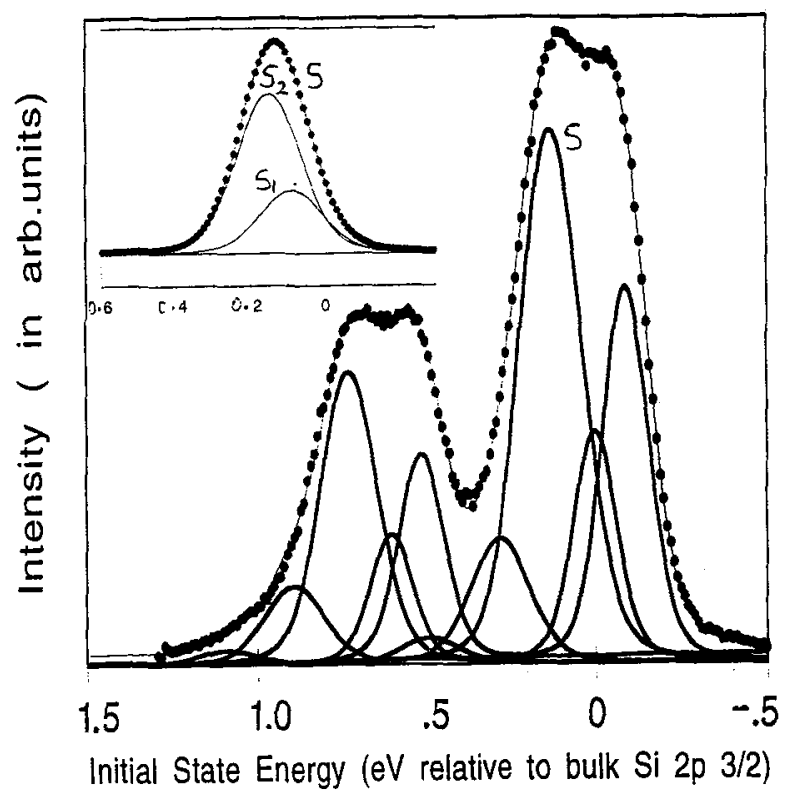

Fig.5: Surface sensitive Si 2p spectrum taken at $\mathrm{hv}=130 \mathrm{eV}$ at $300 \mathrm{~K}$ for $45^{\circ}$ emission angle where the bulk and surface contribution are clearly resolved. The insert shows the splitting of the $2 \mathrm{p} 3 / 2 \mathrm{Si} / \mathrm{H}$ surface component suggested by Blase et al.

The result of the deconvolution together with the calculated values of Blase et al. are summarized in Table 1. The best fit of the bulk component is obtained for a lorentzian FWHM ( lifetime broadening ) of $35 \mathrm{meV}$ [1]. We can see in this table that all the predicted core level shifts fit with the components extracted from the deconvolution apart from a overall shift of $40 \mathrm{meV}$ probably related to the finite size of the silicon slab used for the calculations. However, a satisfactory fit in the entire energy range scanned can only be obtained when introducing two additional components, located at $300 \mathrm{meV}$ and $500 \mathrm{meV}$ relative to the bulk component. These components are not predicted by the theory which estimates the contribution of the third and fourth silicon layer to be negligible. The later argument is in fact the reason why we do not add any other component related to the third and fourth $\mathrm{Si}$ atoms anymore[1]. 


\begin{tabular}{|c|c|c|c|}
\hline & & \\
\hline & & Calculated [4] & Experimental \\
\hline \multirow{6}{*}{$\begin{array}{l}\text { Position relative to } \\
\text { the bulk component }\end{array}$} & Backbonds & -116 & $-88(+/-5)$ \\
\hline & $S 13 / 2$ & 44 & $80(+1-5)$ \\
\hline & $S 23 / 2$ & 114 & $150(+/-5)$ \\
\hline & $\mathrm{S} 1 / 2$ & $80 *$ & $120(+/-5)$ \\
\hline & S3 & - & $300(+/-10)$ \\
\hline & $S 4$ & - & $500(+/-20)$ \\
\hline \multirow[b]{2}{*}{ Phonon broadening } & Surface & - & $\begin{array}{l}175(\text { at } 300 \mathrm{~K}) \\
140 \text { (at } 100 \mathrm{~K})\end{array}$ \\
\hline & Bulk & - & $\begin{array}{l}125(\text { at } 300 \mathrm{~K}) \\
110(\text { at } 100 \mathrm{~K})\end{array}$ \\
\hline Lifetime broadening & . & - & $35(+/-5)$ \\
\hline
\end{tabular}

Table 1: Comparison between recent theoretical value for the position of the core level components [4]) and experimental values obtained following the curve fitting procedure described in the text. All energies are in meV.

*: from X.Blase and Xuejun Zhu private communication.

Since the surface is perfectly hydrogen terminated, one could expect some contribution from the two vibrational motions of the Si-H bonds ( stretching and bending modes), as recently observed in the gas phase[11]. It is difficult to predict whether these vibrational features contribute mainly to the intensities of the components extracted from the fit, the origin of the two highest binding energy contributions remains thus rather uncertain.

In contrast to the valence band region, no significant difference is observed in the core levels for the dihydride terminated stepped $\mathrm{H} / \mathrm{Si}(111)$ surfaces in comparison with the flat surfaces. This indicates that long range ordered defects do not induce either an additional shift or a significant broadening of the core levels.

\section{Conclusion}

Chemically prepared, long range ordered, ideally hydrogen terminated surfaces are model systems for the study of the electronic properties of silicon surfaces. The long range order of these surfaces leads to very sharp features in the valence band and core level spectra which allows a precise definition of the surface states.

The position and relative intensity of the $\mathrm{Si} 2 \mathrm{p}$ core level components asssociated to the top silicon atoms and the subsurface atoms at the $\mathrm{H} / \mathrm{Si}(111)$ surface are in very good agreement with the new theoretical values obtained by X. Blase et al.[4]. 
No important broadening is observed in the room temperature core level and valence band spectra of dihydride terminated stepped $\mathrm{H} / \mathrm{Si}(111)$ surfaces in comparison to the flat ones, in contrast with more inhomogeneous Si surfaces as the $7 \times 7$ reconstructed surface for example.

However, an additional surface state below the Fermi level is observed in the valence band spectra of dihydride terminated stepped silicon surfaces, which is tentatively assigned to the lattice deformation induced by the dihydride steps.

\section{References}

[1]-Hricovini K., Günther R., Thiry P., Taleb-Ibrahimi A., Indlekofer G., Bonnet J.E., Dumas P., Petroff Y., Blase X., Xuejun Zhu, Louie S.G., Chabal Y.J. and Thiry P.A., Phys. Rev. Lett. 70 (1993) 1992.

[2]-Higashi G.S., Chabal Y.J., Trucks G.W. and Raghavachari K., Appl. Phys. Lett. 56 (1990) 656.

[3]-Dumas P., Chabal Y.J. and Higashi G.S., Phys. Rev. Lett. 65 (1990) 1124; Dumas P. and Chabal Y.J., Chem. Phys. Lett. 181 (1991) 537.

[4]-Blase X., Da Silva A.J.R., Xuejun Zhu, and Louie S.G., to be published.

[5]-Jakob P. and Chabal Y.J., J. Chem. Phys. 95 (1991) 2897; Jakob R., Chabal Y.J., Raghavachari K., Becker R.S. and Becker A.J., Surf. Sci., 275 (1992) 407.

[6]-Karisson C.J. et al, Phys. Rev. B 41 (1990) 1521.

[7]-Landemark E., Karlsson C.J. and Uhrberg R.I.G. Phys. Rev. B 44 (1991) 1950.

[8]-Günther R., Taleb-Ibrahimi A, Dumas P., Indlekofer G., Chabal Y.J. and Petroff Y. to be published.

[9]-Raghavachari K., Jakob P. and Chabal Y.J. Chem. Phys. Lett. 206 (1993) 156.

[10]-Hines M.A., Harris T.D., Harris A.L. and Chabal Y.J., J.Electron Spectros. Related Phenomena 64/65 (1993) 183.

[11]-Bancroft G.M. et al, in Abstracts of VUV10 Conference, Paris July 1992. 